\title{
MEDIÇÕES INSTRUMENTAIS E SENSORIAIS DE DUREZA E SUCULÊNCIA NA CARNE CAPRINA ${ }^{1}$
}

\author{
Ângela da Silva BORGES ${ }^{2}$, Jorge Fernando Fuentes ZAPATA $^{2, *}$, Deborah dos Santos GARRUTI ${ }^{3}$, \\ Maria do Carmo Passos RODRIGUES ${ }^{2}$, Ednardo Rodrigues FREITAS ${ }^{2}$, Ana Lucia Fernandes PEREIRA ${ }^{2}$
}

\section{RESUMO}

Foi avaliado o efeito do tipo de músculo e da maturação sobre algumas propriedades funcionais e sensoriais da carne caprina. Utilizaram-se os músculos longissimus dorsi, semimembranosus e biceps femoris de cabras com aproximadamente 20 meses de idade. A carne, sem maturar e maturada por sete dias, foi avaliada para perdas por cocção (PPC) e força de cisalhamento (FC), por métodos instrumentais, e para dureza sensorial (DS) e suculência sensorial (SS), por provadores treinados. As PPC não sofreram efeito significativo ( $>$ > 0,05) do tipo de músculo e da maturação da carne. A carne sem maturar do músculo semimembranosus apresentou maior FC que aquelas dos músculos longissimus dorsi e biceps femoris. Em relação ao tipo de músculo, após a maturação, as carnes dos músculos semimembranosus e biceps femoris se apresentaram mais macias que a do longissimus dorsi. Quanto ao efeito da maturação, a FC da carne do músculo semimembranosus diminuiu significativamente. A FC apresentou correlação positiva com DS e correlação negativa com SS. Dureza e suculência avaliadas de forma sensorial apresentaram correlação negativa. A maturação por 7 dias diminuiu a dureza, mas não afetou as PPC da carne dos músculos semimembranosus e bíceps femoris.

Palavras-chave: maturação, perdas por cocção, força de cisalhamento, avaliação sensorial.

\section{SUMMARY}

INSTRUMENTAL AND SENSORIAL ASSESSMENT OF TENDERNESS AND JUICINESS IN GOAT MEAT. The effect of muscle type and aging for seven days on certain functional and sensory properties of goat meat was evaluated. The experiment used meat from longissimus dorsi, semimembranosus and biceps femoris muscles from female goats that were approximately 20 months. After $24 \mathrm{~h}$ of being slaughtered and seven days, the aging meat was instrumentally analyzed for cooking losses (CL) and shear force (SF), as well as for sensory firmness $(\mathrm{F})$ and juiciness $(\mathrm{J})$ by a trained panel. CL was not affected $(\mathrm{p}>0.05$ ) by the type of muscle and aging of the meat. Before aging, meat from semimembranosus muscles showed higher SF than those from longissimus dorsi or bicep femoris muscles. After aging, meat from semimembranosus and biceps femoris muscles were tenderer than those from longissimus dorsi muscle. Meat from the semimembranosus muscle decreased in firmness during aging. Shear force values in meat showed positive correlation with firmness and negative correlation with juiciness as evaluated by the panelists. A negative correlation between firmness and juiciness was observed. Aging of the meat over seven days decreased firmness in goat meat from muscles, but did not affect cooking losses.

Keywords: aging, cooking losses, shear force, sensory evaluation.

\section{1 - INTRODUÇÃO}

A carne caprina apresenta um grande potencial mercadológico, pois o consumidor preocupado com os efeitos de saúde dos alimentos tende a valorizar seu baixo conteúdo de gordura em relação a outras carnes vermelhas [15]. Apesar da carne caprina ser considerada de pior palatabilidade que a de bovinos, suínos e ovinos, a maturação parece ser uma boa alternativa para esse problema, [14] porque, além de melhorar a maciez, o tratamento propicia, de acordo com FERNANDES [10], melhor sabor às carnes. O aumento da maciez da carne durante a maturação não se deve exclusivamente à proteólise. A proporção relativa de actina e miosina num estado de ligação mais fraco ou mais forte pode afetar esta propriedade da carne [4].

Historicamente, os métodos instrumentais e os testes sensoriais com provadores treinados têm sido usados pelos

\footnotetext{
${ }^{1}$ Recebido para publicação em 27/3/2006. Aceito para publicação em 20/10/2006 (001708)

${ }^{2}$ Departamento de Tecnologia de Alimentos,

Universidade Federal do Ceará, Fortaleza (CE), Brasil,

E-mail:zapata@ufc.br

${ }^{3} E M B R A P A$, Agroindústria Tropical, Fortaleza, CE, Brasil

* A quem a correspondência deve ser enviada
}

pesquisadores em ciência da carne para determinar diferenças de maciez entre as amostras [31]. Contudo, a indústria da carne tem como prioridade o desenvolvimento de métodos instrumentais para medir na carcaça parâmetros que possam predizer, com precisão, a maciez da carne cozida [32]. De acordo com esses autores, o único método instrumental eficiente é a resistência ao corte da carne cozida, também expressa como força de cisalhamento.

A avaliação sensorial da maciez da carne depende de fatores tais como: tipo de julgador (treinado, semitreinado ou consumidor), método de cozimento, forma de preparação das amostras e tipo de músculo utilizado [22].

A maciez da carne é provavelmente a característica mais estudada quando a preocupação é o consumidor. O segundo atributo de textura em importância parece ser a suculência [6]. O consumidor utiliza os atributos de textura para determinar a qualidade e a aceitabilidade da carne, e a melhor qualidade é expressa em termos de maior maciez e maior suculência.

O músculo longissimus dorsi bovino tem sido utilizado, freqüentemente, como indicador da maciez da carne dos outros músculos da carcaça. Ultimamente, porém, a medi- 
ção da força de cisalhamento nos músculos biceps femoris e semimembranosus tem sido considerada mais adequada para estimar a dureza da carne bovina [28].

Para a avaliação sensorial de textura, as amostras de carne devem ser preparadas da forma mais uniforme possível para minimizar diferenças entre as repetições. Isto inclui a aleatorização da apresentação das amostras, logo após o preparo, para minimizar a influência da ordem das amostras sobre o provador e a instrução adequada para coletar respostas consistentes [3].

A coleta de dados precisos e confiáveis, por outro lado, requer treinamento e calibração do grupo de provadores, definição da terminologia, desenho experimental adequado e controle das condições de preparação de amostras e condução dos testes com os provadores [19].

Vários estudos têm sido realizados com o objetivo de medir a textura por parâmetros físicos, os quais podem ser comparados com análises feitas por julgadores selecionados e treinados [16]. O método físico de medir a força de cisalhamento por meio de uma lâmina de Warner-Bratzler tem sido bastante utilizado e, quando comparado à análise sensorial, é possível encontrar uma correlação alta e significativa em carne bovina [20, 27].

Este trabalho teve como objetivo, portanto, verificar o efeito do tipo de músculo e da maturação sobre as características de dureza e suculência da carne de caprinos tropicais e a correlação entre as medições instrumentais e sensoriais dessas características.

\section{2 - MATERIAIS E MÉTODOS}

Foram utilizadas carnes de seis animais caprinos fêmeas do cruzamento 1/2 Boer x 1/2 SRD (Sem Raça Definida), com idade média de 20 meses, abatidos em estabelecimento industrial segundo os métodos recomendados pelo Regulamento de Inspeção Industrial Sanitária de Produtos de Origem Animal [5].

As carcaças foram divididas em duas metades e coletadas as pernas e os lombos para a retirada dos músculos biceps femoris e semimembranosus da perna e longissimus dorsi do lombo. Os músculos das meias carcaças esquerdas foram utilizados imediatamente (carne não maturada) para as medições de perdas por cocção, força de cisalhamento e análise sensorial de dureza e suculência das carnes. Os músculos das meias carcaças direitas foram embalados a vácuo em filmes de nylon-polietileno e acondicionados à temperatura de refrigeração $\left(2{ }^{\circ} \mathrm{C}\right)$ por sete dias (carne maturada), antes de serem submetidos às mesmas análises descritas para a carne não maturada.

As perdas por cocção (PPC) foram medidas segundo a técnica de ABULARACH, ROCHA \& FELÍCIO [1], sendo utilizadas três fatias de carne, cada uma com aproximadamente 2,0 $\mathrm{cm}$ de espessura, 4,0 $\mathrm{cm}$ de comprimento e $2,5 \mathrm{~cm}$ de largura. As amostras foram pesadas em balança semi-analítica e em seguida, cozidas em forno pré-aquecido a $170{ }^{\circ} \mathrm{C}$, até que a temperatura no centro geométrico atin- gisse $71^{\circ} \mathrm{C}$. Em seguida, foram resfriadas à temperatura ambiente e novamente pesadas, sendo expressa a perda de peso de forma percentual.

A força de cisalhamento ( $F C$ ) foi medida segundo a técnica de ABULARACH, ROCHA \& FELÍCIO [1]. Após a medição de PPC, as fatias de carne cozida foram resfriadas à temperatura ambiente e delas retirados dois cilindros, no sentido das fibras, com o auxilio de um vazador de $1,27 \mathrm{~cm}$ de diâmetro. A força necessária para cortar transversalmente cada cilindro foi medida por um texturômetro TA-XT2 (Stable Micro System, Surrey, England), provido de uma lâmina de Warner-Bratzler, operando com velocidade de $20 \mathrm{~cm} / \mathrm{min}$. A média da força de cisalhamento dos cilindros representou o valor da dureza de cada amostra.

Os atributos sensoriais de dureza e suculência, escolhidos para os estudos de correlação com os dados instrumentais foram avaliados mediante a metodologia de Análise Descritiva Quantitativa proposta por STONE et al. [30].

Para a análise sensorial, foi formada uma equipe treinada seguindo-se um procedimento de recrutamento, préseleção, treinamento e avaliação do desempenho individual dos julgadores (seleção final).

Inicialmente foram recrutados vinte indivíduos por meio de questionário, segundo MEILGAARD, CIVILLE \& CARR [18], para avaliar a afinidade face ao produto, disponibilidade de tempo, interesse de participar dos testes e outros parâmetros ligados à saúde e hábitos alimentares. Os candidatos foram, em seguida, avaliados quanto à habilidade em discriminar diferenças mediante uma série de testes triangulares [13,2] com diferentes amostras de carne, usando-se a Análise Seqüencial de Waald [11, 24] com os parâmetros $\mathrm{p}_{0}=0,35 ; \mathrm{p}_{1}=0,65 ; \alpha=0,05 ; \beta=0,05$.

O treinamento foi realizado em 5 sessões abertas ( $45 \mathrm{~min} /$ sessão) com os 14 provadores selecionados, que consensualmente definiram termos de referências para as escalas para dureza e suculência. A dureza ficou definida como sendo "a força necessária para comprimir um pedaço de carne entre os dentes molares na primeira mordida" e a suculência como “a percepção da quantidade de líquido liberado da amostra na boca após a quinta mastigada". Foi escolhida uma escala linear não-estruturada com os termos de intensidade "pouca" e "muita" ancorados nas extremidades. Foram também escolhidas amostras-referência para esses extremos da escala, sendo o corte filé mignon bovino para pouca dureza e muita suculência, a carne do peito bovino para muita dureza e o corte lagarto para pouca suculência.

A seleção final dos provadores constou de um teste piloto das próprias amostras do experimento, em duas repetições. Os dados de cada provador foram submetidos à análise de variância para os descritores dureza e suculência, tendo como fontes de variação amostras e repetições. Para compor a equipe descritiva final foram selecionados nove provadores os quais apresentaram bom poder discriminativo ( $\mathrm{p}_{\text {amostra }}<0,30$ ); boa reprodutibilidade nos julgamentos $\left(\mathrm{p}_{\text {repeticăo }}>0,05\right)$, e consenso com os demais membros do grupo [7]. 
Para a análise sensorial, as amostras de carne caprina foram preparadas em cubos de aproximadamente $2,0 \mathrm{~cm}$ de aresta, os quais foram submetidos a tratamento térmico em forno pré-aquecido a $170{ }^{\circ} \mathrm{C}$ até atingirem a temperatura de $71{ }^{\circ} \mathrm{C}$ no centro geométrico. Os cubos de carne cozida foram servidos a cada julgador em béqueres de vidro codificados e mantidos em aquecedores à temperatura de $50{ }^{\circ} \mathrm{C}$ até $\mathrm{o}$ momento da avaliação pelos julgadores, juntamente com a ficha de avaliação.

O delineamento experimental foi inteiramente casualizado com seis tratamentos e seis repetições por tratamento. Os tratamentos foram obtidos segundo um esquema fatorial $3 \times 2$, e os fatores analisados foram três tipos de músculos e dois tempos de maturação da carne.

Os valores de dureza e suculência atribuídos pelos julgadores treinados para cada músculo em cada tempo de armazenamento foram utilizados para compor o valor médio considerado nas análises.A análise estatística dos dados foi realizada utilizando-se o programa estatístico Statistical Analysis System - SAS - [23] por meio do procedimento PROC ANOVA para um modelo fatorial. A comparação das médias dos tratamentos foi realizada pelo Teste de Tukey, ao nível de 5\% de significância.

O procedimento PROC CORR [23] foi utilizado para verificar a correlação entre as variáveis sensoriais (dureza e suculência) e as variáveis instrumentais (perdas por cocção e força de cisalhamento).

Em função das correlações significativas, procedeu-se à análise de regressão linear simples e múltipla, utilizandose o Método Stepwise de Eliminação Indireta (Backward) por intermédio do SAS [23], para obter equações de predição da dureza e suculência por meio das determinações instrumentais.

\section{3 - RESULTADOS E DISCUSSÃO}

As PPC nas carnes analisadas variaram entre 20,52\% e $24,40 \%$, sem que tenha sido detectado efeito significativo ( $p>0,05)$ do tipo de músculo, da maturação, e da interação entre esses dois efeitos (Tabela 1). GONÇALVES et al. [12] também não observaram efeito do tempo de maturação (14 dias) nas perdas por cocção da carne de animais ovinos do tipo SRD. Os valores reportados neste estudo foram inferiores aos relatados por DHANDA, TAYLOR \& MURRAY [8] que, analisando carne de cabritos mestiços Boer, encontraram valores em torno de $35,4 \%$ de PPC, enquanto ESENBUGA, YANAR \& DAYIOGLU [9] encontraram valores mais próximos dos aqui reportados em carne de cordeiros mestiços provenientes do Peru (26,6\%).

SCHÖNFELDT et al. [25] sugeriram que, devido ao baixo nível de gordura subcutânea, a carne caprina apresenta menor perda de líquido durante o cozimento que a carne ovina. Esses autores também sugeriram que as PPC das carnes provenientes de músculos diferentes estão relacionadas em grande medida com a presença de epimísio, o tecido conectivo que envolve as massas musculares. Neste estudo, porém, a carne utilizada para esta análise foi preparada retirando-se toda a gordura e o tecido conectivo aparentes. As baixas PPC observadas podem, no entanto, estar relacionadas com uma maior firmeza do endomísio e com uma boa capacidade de retenção de água da carne caprina estudada.

TABELA 1 - Perdas por cocção (PPC, \%) da carne não maturada e maturada por 7 dias, proveniente de diferentes músculos de caprinos do cruzamento Boer x SRD.

\begin{tabular}{lccc}
\hline \multicolumn{1}{c}{$\begin{array}{c}\text { Tipo de } \\
\text { músculo }\end{array}$} & \multicolumn{2}{c}{ Maturação da carne } & Médias $^{1}$ \\
& Não maturada & Maturada por $\mathbf{7}$ dias & \\
\hline Longissimus dorsi & 20,52 & 24,40 & $\mathbf{2 2 , 4 6}^{\mathrm{A}}$ \\
Semimembranosus & 23,50 & 22,76 & $\mathbf{2 3 , 1 3}^{\mathrm{A}}$ \\
Bíceps femoris $^{\text {Médias }}{ }^{1}$ & 22,24 & 22,83 & $\mathbf{2 2 , 5 4}^{\mathrm{A}}$ \\
\hline
\end{tabular}

${ }^{1}$ Médias seguidas pela mesma letra maiúscula, nas colunas, e pela mesma letra minúscula nas linhas, não diferem entre si pelo teste de Tukey $(P>0,05)$.

Em relação à dureza da carne, medida como força de cisalhamento (Tabela 2 ), houve efeito significativo $(\mathrm{p}<0,05)$ do tipo de músculo e da maturação, bem como, da interação entre esses fatores, indicando que a maturação influencia de forma diferente a dureza da carne dependendo da procedência muscular. Desta forma, observou-se que a carne não maturada, proveniente do músculo semimembranosus, apresentou o maior ( $\mathrm{p}<0,05$ ) valor de $\mathrm{FC}$ em relação àquelas dos demais músculos que não diferiram $(p>0,05)$ entre si. Entretanto, após a maturação, o maior $(\mathrm{p}<0,05)$ valor de FC foi registrado para a carne do músculo longissimus dorsi. A carne do músculo semimembranosus, por outro lado, apresentou um acentuado incremento de maciez com o tempo de maturação. Resultados similares foram obtidos por KANNAN et al. [14] que reportaram um declínio significativo da força de cisalhamento do músculo semimembranosus ao estudar a carne de cabras espanholas com 4 dias de maturação. Pode-se afirmar, portanto, que, em relação à resistência ao corte, o efeito da maturação foi mais benéfico para a carne da perna que para a do lombo.

Os valores de FC obtidos neste estudo variaram de 9,56 kgf a 16,62 kgf (Tabela 2), sendo superiores aos relatados por DHANDA, TAYLOR \& MURRAY [8] e SEN, SANTRA $\&$ KARIM [26] que encontraram valores de $4,2 \mathrm{~kg}$-f na carne de cabritos mestiços Boer e 7,42 kgf em caprinos nativos da Índia, respectivamente. Considerando que a carne com força de cisalhamento acima de $11 \mathrm{kgf}$ é classificada como dura, entre 8 e $11 \mathrm{kgf}$ como aceitável e abaixo de $8 \mathrm{kgf}$ como macia [29], pode-se estimar que a carne dos animais em estudo se apresentou relativamente dura. Isto pode se dever ao fato dos animais deste estudo terem idade média de abate de 20 meses.

TABELA 2 - Força de cisalhamento (kgf) da carne não maturada e maturada por 7 dias, proveniente de diferentes músculos de caprinos do cruzamento Boer x SRD.

\begin{tabular}{lccc}
\hline \multirow{2}{*}{ Tipo de músculo } & \multicolumn{2}{c}{ Maturação da carne } & Médias $^{1}$ \\
\cline { 2 - 3 } & Não maturada & Maturada por 7 dias & \\
\hline Longissimus dorsi & $13,25^{\mathrm{aB}}$ & $13,36^{\mathrm{aA}}$ & $\mathbf{1 3 , 3 1}$ \\
Semimembranosus & $16,62^{\mathrm{aA}}$ & $10,71^{\mathrm{bB}}$ & 13,67 \\
Bíceps femoris $^{\mathrm{B}}$ & $11,25^{\mathrm{aB}}$ & $9,56^{\mathrm{bB}}$ & 10,41 \\
Médias $^{1}$ & $\mathbf{1 3 , 7 1}$ & $\mathbf{1 1 , 2 1}$ & \\
\hline
\end{tabular}

${ }^{1}$ Médias seguidas pela mesma letra maiúscula, nas colunas, e pela mesma letra minúscula nas linhas, não diferem entre si pelo teste de Tukey $(P>0,05)$. 
Na Tabela 3, são apresentados os valores de dureza sensorial (DS) e suculência sensorial (SS) de carne caprina registrados por provadores treinados. Houve efeito significativo $(\mathrm{p}<0,05)$ do tipo de músculo e da maturação, bem como da interação entre esses fatores, indicando que a maturação influencia de forma diferente a DS e a SS da carne dos músculos analisados.

Quanto ao efeito da maturação, os julgadores encontraram redução na dureza das carnes dos músculos semimembranosus e biceps femoris, enquanto a carne do músculo longissimus dorsi não foi influenciada significativamente ( $\mathrm{p}>0,05)$.

Em relação ao tipo de músculo, observou-se que antes e após a maturação a carne do músculo biceps femoris apresentou-se menos dura que a dos demais músculos estudados, assim como a do músculo semimembranosus em comparação com a do longissimus dorsi. Isto sugere que a maturação favoreceu o amaciamento, percebido sensorialmente, da carne dos músculos semimembranosus e biceps femoris, e não afetou a dureza da carne do músculo longissimus dorsi. Esta constatação também pôde ser verificada observando-se os menores valores de força de cisalhamento (Tabela 2), obtidos para a carne destes dois músculos.

No que se refere à SS, a maturação por sete dias levou a uma redução $(\mathrm{p} \leq 0,05)$ desta característica somente na carne do músculo longissimus dorsi (Tabela 3).

Independentemente do tempo de maturação, a carne do músculo biceps femoris apresentou-se mais suculenta quando comparada a dos músculos longissimus dorsi e semimembranosus, que, por sua vez, diferiram apenas após a maturação, quando a carne do músculo semimembranosus apresentou-se mais suculenta que a do músculo longissimus dorsi. A maior suculência da carne de biceps femoris pode estar associada com a menor força de cisalhamento apresentada por esta carne (Tabela 2).

MADRUGA et al. [17] ao estudarem caprinos mestiços (Crioulo x Anglo Nubiano) observaram valores de suculência $(5,91)$ semelhantes aos deste estudo (Tabela 3$)$. Para o atributo suculência, PONNAMPALAM et al. [21], em ovinos da Austrália, verificaram média de 5,7 e ESENBUGA, YANAR \& DAYIOGLU [9], em cordeiros do Peru, encontraram valor de 5,5, provavelmente devido ao fato desses autores terem trabalhado com animais mais novos que os do presente estudo.

Na Tabela 4, são apresentados os coeficientes de correlação e as respectivas significâncias, entre os fatores de qualidade medidos instrumentalmente e por provadores treinados. A FC apresentou correlação positiva significativa $(\mathrm{r}=0,63, \mathrm{p}<0,05)$ com a DS. Dessa forma, foi observado que à medida que aumenta a $\mathrm{FC}$ a DS da carne também aumenta, enquanto que a SS diminui. A FC, no entanto, não apresentou correlação com as PPC.

Por outro lado, foi verificado que a DS e SS correlacionam-se negativamente $(r=-0,75, p<0,05)$ entre si, indicando que os provadores avaliaram como menos suculentas as carnes mais duras (Tabela 4). Esse fato foi confirmado pela análise instrumental, pois a SS apresentou correlação negativa significativa, embora moderada $(r=-0,35 ; p<0,05)$ com a força de cisalhamento. Esta correlação era esperada, pois, de acordo com MUÑOZ \& CHAMBERS [19], a análise descritiva pode fornecer a informação técnica, precisa e confiável necessária sobre os atributos de qualidade sensorial do alimento.

TABELA 4 - Correlação entre os valores de força de cisalhamento (FC), perdas por cocção (PPC), dureza sensorial (DS) e suculência sensorial (SS) da carne caprina (Cruzamento Bôer x SRD).

\begin{tabular}{ccccc}
\hline & FC & PPC & DS & SS \\
\hline FC & 1,00000 & $0,13791^{\text {ns }}$ & $0,63381^{\text {** }}$ & $-0,35485^{*}$ \\
PPC & - & 1,00000 & $0,04153^{\text {ns }}$ & $-0,17284^{\text {ns }}$ \\
DS & - & - & 1,00000 & $-0,75105^{* *}$ \\
SS & - & - & - & 1,00000 \\
\hline n.s. $=$ não significativo $(\mathrm{p}>0,05) ;{ }^{*}=$ significativo $(\mathrm{p}<0,05) ; \mathrm{e}^{* *}=$ significativo
\end{tabular}
$(\mathrm{p}=0,01)$

Na tentativa de se obter uma equação para estimar os valores de dureza e suculência das carnes caprinas em função das análises instrumentais, observou-se que esses atributos sensoriais podem ser estimados em função da força de cisalhamento segundo as equações:

$D S=-1,96+0,50 F C\left(R^{2}=0,40\right)$

$\mathrm{SS}=6,94-0,15 \mathrm{FC}\left(\mathrm{R}^{2}=0,13\right)$

Apesar dos baixos coeficientes de correlação entre as medições observados neste estudo com carne caprina, eles foram significativos ( $\mathrm{p}<0,05)$. Em estudos com carne bovina do músculo longissimus, no entanto, têm sido reportadas correlações altas $(r=0,92)$ entre a maciez instrumental, medida pela força de cisalhamento, e a maciez sensorial, medida por um painel de consumidores [32].

\section{4 - CONCLUSÕES}

A maturação por sete dias favorece a maciez da carne caprina dos músculos da perna semimembranosus e biceps

TABELA 3 - Valores atribuídos à dureza e suculência sensoriais da carne não maturada e maturada por 7 dias, proveniente de diferentes músculos de caprinos do cruzamento Boer x SRD.

\begin{tabular}{|c|c|c|c|c|c|c|}
\hline \multirow{3}{*}{$\begin{array}{l}\text { Atributo } \\
\text { sensorial }\end{array}$} & \multicolumn{6}{|c|}{ Tipo de músculo } \\
\hline & \multicolumn{2}{|c|}{ Longissimus dorsi } & \multicolumn{2}{|c|}{ Semimembranosus } & \multicolumn{2}{|c|}{ Biceps femoris } \\
\hline & Não maturada & Maturada por 7 dias & Não maturada & Maturada por 7 dias & Não maturada & Maturada por 7 dias \\
\hline Dureza $^{1}$ & $6,43^{\mathrm{aA}}$ & $6,41^{\mathrm{aA}}$ & $5,20^{A b}$ & $3,77^{\mathrm{bB}}$ & $2,25^{\mathrm{aC}}$ & $1,34^{\text {bC }}$ \\
\hline Suculência² & $4,89^{\mathrm{aB}}$ & $3,05^{\mathrm{bc}}$ & $5,00^{A b}$ & $4,80^{\mathrm{aB}}$ & $6,01^{\mathrm{aA}}$ & $6,27^{\mathrm{aA}}$ \\
\hline
\end{tabular}


femoris, mas não afeta a carne do músculo longissimus dorsi do lombo.

O tipo de músculo influencia a dureza analisada de forma instrumental, assim como a dureza e a suculência da carne, analisadas por julgadores treinados.

A maturação por sete dias e o tipo de músculo não influenciam as perdas por cocção da carne caprina.

A medição instrumental da dureza da carne (força de cisalhamento) pode ser usada como uma estimativa da dureza sensorial da carne caprina.

\section{5 - REFERÊNCIAS BIBLIOGRÁFICAS}

[1] ABULARACH, M. L. S.; ROCHA, C. E.; FELICIO, P. E. de. Características de qualidade do contrafilé (m. L. dorsi) de touros jovens da raça Nelore. Ciência e Tecnologia de Alimentos, Campinas, v. 18, n. 2, p. 205-210, 1998.

[2] AMERICAN SOCIETY FOR TESTING AND MATERIALSASTM. Guidelines for the selection and training of sensory panel members. Philadelphia: ASTM, 1981. $758 \mathrm{p}$.

[3] BET, K. L. Measuring sensory properties of meat in the laboratory. Food Technology, Chicago, v. 47, n. 11, p. 121-126, 1993 .

[4] BOND, J. J.; CAN, L. A.; WARNER, R. D. The effect of exercise stress, adrenaline injection electrical stimulation on changes in quality attributes and proteins in semimembranosus muscle of lamb. Meat Science, Barking, v. 68, n. 3, p. 469-477, 2004.

[5] BRASIL. Ministério da Agricultura. Regulamento da Inspeção Industrial e Sanitária de Produtos de Origem Animal - RIISPOA. Brasília-DF: Ministério da Agricultura, 1997. 85 p.

[6] CHAMBERS, E. IV.; BOWERS, J. R. Consumer perception of sensory qualities in muscle foods. Food Technology, Chicago, v. 47, n. 11, p. 116-120, 1993.

[7] DAMASIO, M. H.; COSTELL, E. Descriptive sensory analysis - generation of descriptors and selection of judges. Revista de Agroquímica y Tecnologia de Alimentos, Valencia, v. 31, n. 2, p. 165-178, 1991.

[8] DHANDA, J. S.; TAYLOR, D. G.; MURRAY, M. J. Part 1. Growth, carcass and meat quality parameters of male goats: effects of genotype and live weight at slaughter. Small Ruminant Research, Amsterdam, v. 50, n. 1, p. 57-66, 2003.

[9] ESENBUGA, N.; YANAR, M.; DAYIOGLU, H. Physical, chemical and organoleptic properties of ram lamb carcasses from four fat-tailed genotypes. Small Ruminant Research, Amsterdam, v. 39, n. 2, p. 99-105, 2001.

[10] FERNANDES, J. R. A maturação da carne bovina. In: SEMINÁRIO E WORKSHOP - "PRESERVAÇÃO E ACONDICIONAMENTO DE CARNE BOVINA IN NATURA", 1997, Campinas. Campinas: ITAL, 1997. p. 47-55.

[11] GARRUTI, R. S. Metodologia na seleção seqüencial e não seqüencial para equipes de julgadores. 1976. 211 f. Tese (Doutorado em Ciências de Alimentos)- Faculdade de Engenharia de Alimentos e Agrícola, Universidade Estadual de Campinas, Campinas, 1976.
[12] GONCALVES, L. A. G. et al. Efeito do sexo e tempo de maturação sobre a qualidade da carne ovina. Ciência e Tecnologia de Alimentos, Campinas, v. 24, n. 3, p. 459-467, 2004.

[13] HELM, E.; TROLLE, B. Selection of a taste panel. Wallerstein Laboratories Communications, New York, v. 9, n. 28, p. 181-194, 1946 .

[14] KANNAN, G. et al. Influence of packaging method and storage time on share value and mechanical strength of intramuscular connective tissue of chevon. Journal of Animal Science, Champaign, v. 80, n. 9, p. 2383-2389, 2002.

[15] KANNAN, G.; KOUAKOU, B.; GELAYE, S. Color changes reflecting myoglobin and lipid oxidation in chevon cuts during refrigerated display. Small Ruminant Research, Amsterdam, v. 42, n. 1, p. 67-75, 2001.

[16] LAWRIE, R. A. Ciência da Carne. 6 ed. Porto Alegre: Artmed, 2005. 384 p.

[17] MADRUGA, M. S. et al. Castration and slaughter age effects on panel assessment and aroma compounds of the "mestiço" goat meat. Meat Science, Barking, v. 56, n. 2, p. 117-125, 2000.

[18] MEILGAARD, M.R.; CIVILLE, G.V.; CARR, B.T. Sensory evaluation techniques, Boca Raton: CRC Press, 1987, v. 2, $159 \mathrm{p}$.

[19] MUÑOZ, A. M.; CHAMBERS, E. IV. Relating sensory measurements to consumer acceptance of meat products. Food Technology, Chicago, v. 7, n. 11, p. 128-134, 1993.

[20] OTREMBA, M. M. et al. Interrelationships among evaluations of beef longissimus and semitendinosus muscle tenderness by Warner-Bratzler shear force, a descriptive texture profile sensory panel, and a descriptive attribute sensory panel. Journal of Animal Science, Champaign, v. 77, n. 4, p. 865-873, 1999.

[21] PONNAMPALAM, E. N. et al. Dietary manipulation of muscle long-chain omega- 3 and omega- 6 fatty acids and sensory properties of lamb meat. Meat Science, Barking, v. 60, n. 2, p. 125-132, 2002.

[22] RESURRECCION, A. V. A. Sensory aspects of consumer choices for meat and meat products. Meat Science, Barking, v. 66, n. 1, p. 11-20, 2003.

[23] STATISTICAL ANALISYS SISTEM - SAS. SAS / STAT: Users guide. Version 6, 12 ed. Cary: SAS Institute Inc., 2000.

[24] SHIROSE, I. Análise seqüencial de Wald e sua aplicação à seleção de julgadores para avaliação organoléptica. Boletim do ITAL, Campinas, v. 50, março/abril, p. 57-77, 1977.

[25] SCHÖNFELDT, H. C. et al. Cooking and juiciness-related quality characteristics of goat and sheep meat. Meat Science, Barking, v. 34, n. 3, p. 381-394, 1993.

[26] SEN, A. R.; SANTRA, A.; KARIM, S. A. Carcass yield, composition and meat quality attributes of sheep and goat under semiarid condition. Meat Science, Barking, v. 66, n. 4 , p. 757-763, 2004.

[27] SHACKELFORD, S. D.; WHEELER, T. L.; KOOHMARAIE, M. Repeatability of tenderness measurements in beef round muscles. Journal of Animal Science, Champaign, v. 75, n. 9, p. 2411-2416, 1997. 
[28] SIMÕES, J. A.; MENDES, M. I.; LEMOS, J. P. C. Selection of muscles as indicators of tenderness after seven days of ageing. Meat Science, Barking, v. 69, n. 4, p. 617-620, 2005

[29] SOUZA, X. R. et al. Efeitos do grupo genético, sexo e peso ao abate sobre as propriedades físico-químicas da carne de cordeiros em crescimento. Ciência e Tecnologia de Alimentos, Campinas, v. 24, n. 4, p. 543-549, 2004

[30] STONE, H. S. et al. Sensory evaluation by quantitative descriptive analysis. Food Technology, Chicago, v. 28, n. 11 , p. 24-34, 1974

[31] WHEELER, T. L., SHACKELFORD, S. D., KOHMARAIE, $M$. The accuracy and repeatibility of untrained laboratory consumer panelists in detecting differences in beef longissimus tenderness. Journal of Animal Science, Champaign, v. 82, n. 2, p. 557-562, 2004.

[32] WHEELER, T. L., VOTE, D., LEHESKA, J. M., SHACKELFORD, S. D., BELK, K. E., WULF, D. M., GWARTNEY, B. L., KOHMARAIE, M. The efficacy of three objective systems for identifying beef cuts that can be guaranteed tender. Journal of Animal Science, Champaign, v. 80, n. 12, p. 3315-3327, 2002.

\section{6 - AGRADECIMENTOS}

À FUNCAP, Fundação Cearense de Amparo à Pesquisa, pela bolsa de mestrado concedida à primeira autora. Ao CNPq, pelo apoio financeiro ao projeto. À Embrapa Agroindústria Tropical, pela colaboração nas análises instrumentais e sensoriais da carne. Aos provadores, pela colaboração e dedicação nos testes sensoriais. 